\title{
EFFECT OF SOME SOIL AMENDMENTS AND COMPOST ON SOME SOYBEAN VARIETIES PRODUCTIVITY AND SOIL PROPERTIES UNDER SALT AFFECTED SOILS \\ Atwa, A. A. $E^{\star}$ and R. A. I. Abo Mustafa** \\ * Soils, Water and Environment Research Institute, Agric. Res. Center \\ **Field Crops Research Institute, (A. R .C)
}

\begin{abstract}
A field experiment was conducted during the two successive summer seasons of 2012 and 2013 at the experimental farm of Sakha Agric. Res. Station, Kafr EL-Sheikh Governorate. This study was conducted to investigate the effects of gypsum, sulphur and organic fertilizer (rice straw compost) on soybean yield, its chemical composition and soil chemical properties under three levels of soil salinity5.00, 9.00and $13.00 \mathrm{dS} / \mathrm{m}$. The experiments were laid in split plot design, where the main plots, were allocoted for soil amendments: control treatment,-Rice straw compost at rate of 4 ton fed $^{-1}$, sulphur at rate of $800 \mathrm{~kg}$ fed and gypsum at level of 4 tonfed $^{-1}$ while the sub plots were allotted for soybean cultivars; Giza111, Dr 101, Giza35, Giza82, Giza83, Toano and Holyday. The treatments were replicated four times.

The results can be summarized as follows:-

1 - The soybean yield and its components were significantly affected by salinity, soil amendments and soybean cultivars

2- The highest soybean seed yield were obtained by Giza 35 and Giza83 under application of gypsum treatment at three levels of soil salinity

3- The maximum values of $\mathrm{N}, \mathrm{P}$ and $\mathrm{K}$ contents in the seeds were obtained by Giza111 .Giza 35 andGiza82 under gypsum treatment

4- The highest straw yields were recorded from Toano and Holyday, cultivars

5 -Soil salinity decreased as follows gypsum $>$ sulphur>compost>control in the soil at the end of experiments

6- Availability of N, P and K of soil after harvesting of soybean plants were increased at S1and S2 especially with application of compost comparing to control

7-The seed yield as affected by cultivars which can be arranged according to tolerance for salinity as follows: Giza83 = Giza35= Giza111=Dr101= Holyday $=$. Toano > Giza 82) at S1. (Giza83 = Giza35> Giza111. =Dr101= Holyday = Toano > Giza82) at s2 and Giza83 = Giza35> Giza111. =Dr101> Holyday = Toano > Giza82) at S3 under gypsum treatment respectively
\end{abstract}

\section{INTRODUCTION}

Soybean (Glycine Max L.) a healthy food, is a cheap source of oil and protein. Sulphur considered of special importance for oil plants due to its essentiality in amino and nucleic acids formation and protein metabolism (Mohamed et al, 2001). Sulphur reduces soil pH resulting in higher nutrient availability and better physical conditions (Agrisnet: Manures, 2011). Therefore the regular addition of compost is the best way for enhancing soil organic and, which helps to build a fertile soil structure. Such a soil structure makes better use of water and nutrients. It easier to till and, overall, is better able to achieve optimum yields on a long term basis(Rangarajan et al., 2000) 
demonstrated through a filed study that organic fertilizers significantly increased $\mathrm{N}$ and $\mathrm{K}$ uptake and yield of legume crop. Application of gypsum is a common recommendation as a source of calcium to replace exchangeable sodium and to reduce alkalinity and improve physical and chemical properties of the soil (Ayers and Westcot, 1985). In the Nkwalini, Various amendments like gypsum, sulphur, acids, press mud and farm yard manure (FYM) may be used for reclamation of these soils (Muhammad 1990,Sharma et al. 1996, Biggar 1996, Haq et al., 2001). The use of gypsum as a source of $\mathrm{Ca}_{2}+$ is a well established practice for the amelioration and management of sodium saturated water/soils (Bresler, et al., 1982). Being easily available and cheap source of calcium, gypsum is commonly used in Pakistan .Because of low solubility of gypsum and calcareous nature of soils its efficiency is reduced. However, its effect on the amelioration process continues for few months until the whole of gypsum reacts with the exchangeable sodium $(\mathrm{Na})$ of the soil.

Soil salinity is one of the most important environmental factors affecting the growth and yield of most field crops, especially in arid and semi-arid regions as in Egypt. Saline soil is wide-spread in the northern part of the country especially in Kafr El-Sheikh Governorate. The problem of salinity received much attention in Egypt in both old cultivated and newly reclaimed areas. Effects on growth and yield may be due to ionic imbalances which can be caused by high salt concentration and soluble salts which depress the water potential of nutrient medium and hence restrict water uptake by plant roots. Salinity causes reduction in crop yield on about 10Mha of world irrigated land (Rhoades and Loveday, 1990). One of the major reasons of low productivity of crops grown under saline sodic conditions is the

Salt toxicity. The management of salt affected soil requires a good understanding of crop- salinity relations, particularly under field condition.

It is common that field crop differ greatly in their tolerance to salinity and the differences in salt tolerance often occur between different varieties of a given species. Actual, response to salinity varies also according to stage of plant growth (Jefferies, 1988).

Salinity seriously constrains crop yield in irrigated agriculture throughout the world. Nearly one third of the world's irrigated agricultural land is saline, (Shannon, 1984) and estimates salt-affected soil by about 400-950 $x 10^{6}$ ha. Saline and alkaline soils are the major problem which affects productivity of common bean in arid and semi-arid regions such as Egypt. It has bean generally recorded that salinity adversely affects seed germination and seedling growth as well as relevant metabolic processes of some glycophytic plants (Ahmed et al., 1983; Drossopoulos et al., 1987). As glycophyte (Hasegawa et al., 2000). Salt affected soils are characterized by excessively high levels of water-soluble salts, including sodium chloride ( $\mathrm{NaCl})$, sodium sulfate $\left(\mathrm{Na}_{2} \mathrm{SO}_{4}\right)$, calcium chloride $\left(\mathrm{CaCl}_{2}\right)$ and magnesium chloride $\left(\mathrm{MgCl}_{2}\right)$, among others. The physical, chemical and biological properties of salt affected soil are improved by the application of gypsum and/or FYM as remediation for sustainable land usage and crop productivity, leading to enhanced plant growth and development (Chaudhry, (2001). 


\section{MATERIALS AND METHODS}

A field experiment was carried out at Sakha Agriculture Research Station farm using soybean (Glycine max L.) during the two successive summer seasons of 2012and 2013. The soil of the experimental field was clayey in texture as shown in Tables (2)and (3),some, soil chemical analysis are determined according to Page (1982) before planting 2012 and after harvesting 2013 and physical properties of the soil were determined according to Klute (1986).

Split plot design was assigned by four treatments. The main plot 1control treatment 2-rice straw compost at rate of 4 tonfed $^{-1}, 3$ sulphur at rate of $800 \mathrm{~kg} \mathrm{fed}^{-1} 14$-gypsum at rate of 4 Tonfed $^{-1}$ The sub plots were allotted to seven soybean cultivars, cultivars Giza111, Dr 101, Giza35, Giza82, Giza83, Toano and Holyday. The treatments were replicated four times for each. Each replicate had 7 plots randomly assigned to the 7 genotypes. Each plot consisted of 5 ridges three meters length with $60 \mathrm{~cm}$ between ridges. Sowing took place as to rows/ridge, in double seeded hills, $20 \mathrm{~cm}$ apart. At harvest the mid three ridge/plot were harvested where the plot area was $5.4 \mathrm{~m}^{2}$ All treatments were fertilized with $\mathrm{N}$ fertilizer as urea at level of $15 \mathrm{~kg} \mathrm{Nfed}^{-1}$, super phosphate $\left(22.5 \mathrm{~kg} \mathrm{pfed}^{-1}\right)$ and potassium sulphate $\left(24 \mathrm{~kg} \mathrm{k}_{2} \mathrm{Ofed}^{-1}\right)$ .The other recommended agriculture practices were done.

Table (1): Pedigree origin and growth habit of the studied soybean genotypes

\begin{tabular}{|l|c|c|c|c|}
\hline Cod & Genotypes & Pedigres & origin & Growth habit \\
\hline 1 & Giza 111 & Crawford xcelest & Egypt & Indeterminate \\
\hline 2 & Dr 101 & $\begin{array}{c}\text { Selected from Elgin(f4 selection } \\
\text { from the populationp6) }\end{array}$ & Egypt & Determinate \\
\hline 3 & Giza35 & Crawford xcelest & Egypt & Indeterminate \\
\hline 4 & Giza82 & Crawford x M-presto & Egypt & Indeterminate \\
\hline 5 & Giza83 & Select from MBB-123-9 & Egypt & Indeterminate \\
\hline 6 & Toano & Worex Essax & USA & Determinate \\
\hline 7 & Holyday & N77-179x Johnston & USA & Determinate \\
\hline
\end{tabular}

\section{Studied characters:}

1-Yield and its components:, $9999999 \% 99 \%$ Biological yield, seed yield and .straw yield (ton/fed) Harvest index: seed yield /biological yield and 100 -seed weight $(\mathrm{g})$

2-Some mineral composition of soybean seed: i.e., nitrogen, Phosphorus and potassium were determined according to method introduced by Jackson (1967). Protein percentage was calculated by multiplying the total nitrogen $\%$ by 5.71 according to (FAO/WHO.1973).

3- Salinity, nutrient contents (Available N, P and K) of representative of soil content after soybean harvesting were determined according to the standard methods (Page, 1982) following: The objective of the present work is to investigate the effects of soil amendment sulphur, gypsum and compost on soybean yield and its chemical composition, rather for seed 
contents of $\mathrm{N}, \mathrm{P}$ and $\mathrm{K}$ for sustainable agriculture and soil chemical properties

Table (2): Some Chemical analyses of soil $(0-60 \mathrm{~cm})$ before planting (2012)

\begin{tabular}{|c|c|c|c|c|c|c|c|c|c|c|c|c|c|}
\hline \multirow{2}{*}{ 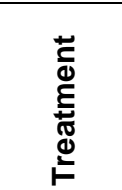 } & \multirow{2}{*}{ 疍 } & \multirow{2}{*}{ Ðூّ } & \multicolumn{4}{|c|}{$\begin{array}{c}\text { Soluble cations, meq } \\
/ L\end{array}$} & \multicolumn{4}{|c|}{$\begin{array}{c}\text { Soluble anions, } \\
\text { meq/L }\end{array}$} & \multirow{2}{*}{ 흉 } & \multicolumn{2}{|c|}{$\begin{array}{c}\text { Available } \\
\text { ppm }\end{array}$} \\
\hline & & & $\mathrm{Ca}^{+2}$ & $\mathbf{M g}^{+2}$ & $\mathrm{Na}^{+}$ & $\mathrm{K}^{+}$ & $\mathrm{CO}_{3}^{-}$ & $\mathrm{HCO}_{3}^{--}$ & $\mathrm{CL}^{-}$ & $\mathrm{SO}_{4}^{--}$ & & P\% & K \\
\hline Gypsum & $\mathrm{S1}$ & 5.32 & 16.1 & 7.2 & 28.02 & 1.96 & . & 10.0 & 14.5 & 28.78 & 0.11 & 0.40 & 290 \\
\hline sulphur & S1 & 5.03 & 15.8 & 7.1 & 25.4 & 2.00 & & 9.8 & 16.0 & 24.50 & 0.11 & 0.40 & 290 \\
\hline control & S1 & 5.13 & 16.0 & 7.2 & 26.1 & 1.99 & & 9.9 & 16.2 & 25.19 & 0.11 & 040 & 290 \\
\hline compost & S1 & 5.58 & 16.1 & 7.3 & 25.2 & 198 & - & 9.8 & 16.1 & 24.68 & 0.11 & 040 & 290 \\
\hline Gypsum & S2 & 9.65 & 30.02 & 19.1 & 45.2 & 2.20 & 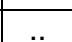 & 13.1 & 41.8 & 41.62 & 0.10 & 035 & 300 \\
\hline sulphur & S2 & 9.94 & 31.0 & 20.1 & 46.2 & 2.10 & 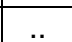 & 14.1 & 42.6 & 42.70 & 0.10 & 035 & 300 \\
\hline control & S2 & 9.84 & 30.1 & 18.2 & 48.1 & 2.10 & & 12.6 & 43.2 & 42.70 & 0.10 & 035 & 300 \\
\hline compost & S2 & 9.64 & 30.2 & 18.0 & 46.1 & 2.10 & .. & 12.8 & 42.2 & 41.40 & 0.10 & 035 & 300 \\
\hline Gypsum & S3 & 13.4 & 36.2 & 22.1 & 72.5 & 3.2 & .. & 13.5 & 68.4 & 52.1 & 0.08 & 0.38 & 338 \\
\hline sulphur & S3 & 13.47 & 36.8 & 23.1 & 71.5 & 3.3 & .. & 15.4 & 68.2 & 51.1 & 0.08 & 0.38 & 338 \\
\hline control & S3 & 13.57 & 36.7 & 23.1 & 72.5 & 3.4 & & 15.2 & 68.1 & 52.4 & 0.08 & 0.38 & 338 \\
\hline compost & S3 & 13.0 & 36.6 & 23.2 & 71.1 & 3.5 & & 15.1 & 68.2 & 46.7 & 0.08 & 0.38 & 338 \\
\hline
\end{tabular}

- Soil under study surrounded by research buildings from three sides and fourth sides was limited by mean drain. So, the drainage was restricted.

\section{RESULTS AND DISCUSSION}

Effect of soil amendment and compost on soil properties after soybean harvesting

Soil salinity:

Effects of different soil amendment treatments with sulphur gypsum and compost on soil salinity comparing control after harvesting soybean plants are shown in Table 3. Data show that addition of gypsum amendments decreased soil salinity from $(5.32,5.03,5.13,5.58),(9.65,9.94,9.84,9.64)$ and $(13.4,13.47,13.57,13.0) \mathrm{dS} / \mathrm{m}$ to $(3.61,4.10,5.06,6.98), \quad(3.47,2.94,7.31,7.22)$ and $(3.39,7.30,7.21,8.95) \mathrm{dS} / \mathrm{m}$ at amendment gypsum, sulphur, control and compost, respectively. This may be due to the dissolving action of replacing calcium place sodium resulted from sulphur oxidation by microbial activity which react with $\mathrm{CaCO}_{3}$ and calcium phosphates and ends up with the formation of $\mathrm{CaSO}_{4}$, total salinity is thus increased. The same conclusions were reported by (Abd-Allahh,1998).

Available soil macronutrients content:

Data in Table (3) revealed that the Soil amendment and compost affects on the availability of $\mathrm{N}, \mathrm{P}$ and $\mathrm{K}$ after harvesting soybean plants.

$\mathrm{N} \%$, available $\mathrm{P}$ and $\mathrm{K}$ content in soil were increased up to $0.11 \% .0 .40 \% .290, .10 .0 \% .35 \% .300$, and $0.09 \% .0 .40 \% .315 \mathrm{mg} / \mathrm{kg}$ before 
planting at $\mathrm{s} 1, \mathrm{~s} 2$ and $\mathrm{s} 3$ in the two seasons respectively to (0.12\%.0.46\%.320), $(0.12 \% .0 .46 \% .320)$ and $(0.13 \% .0 .48 \% .320 \mathrm{mg} / \mathrm{kg})$ at the end of experiments with gypsum .sulphur and compost at s1 and s2.while NPK decreased comparing before planting at s3 because the nodules of soybean affects with soil salinity. It was noticed that soil available nutrients contents resulting from the application of composted rice straw. This may be due to the short time growth period of soybean in which reflect residual of nutrients from, OM decomposition. Thus highly levels were interpreted by many others, Metwally, and Khamis, (1998). Stated that organic maturing plays role in increasing the $\mathrm{N}$ availability through microorganism activity besides decreasing $\mathrm{N}$ losses by leaching and volatilization. The increase in the availability of soluble $\mathrm{P}$ from additions of compost which has an effect that described as resulting from phosphors humic complexes that minimize immobilization processes, anion replacement of phosphate by humid ions, and coating of sequie oxides particles by humus to form a cover which reduces the phosphate fixating capacity Rechcigl, (1995)Concerning the increasing of available $\mathrm{K}^{+}$after addition of compost, Tan (1993) found that humid and fulvic acids are capable for dissolving very small amounts potassium from the soil minerals by chelating complex reaction or both with released amounts of $\mathrm{K}$ being increased by time

Table (3): Some chemical analyses of soil ${ }^{*}(0-60 \mathrm{~cm})$ after end of the experiments

\begin{tabular}{|c|c|c|c|c|c|c|c|c|c|c|c|c|c|}
\hline \multirow{2}{*}{ 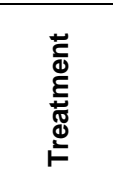 } & \multirow{2}{*}{ 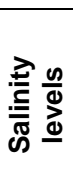 } & \multirow{2}{*}{$\begin{array}{c}E C e \\
d S / \\
m\end{array}$} & \multicolumn{4}{|c|}{ Soluble cations, meq/L } & \multicolumn{4}{|c|}{ Soluble anions, meq/L } & \multirow[b]{2}{*}{$\begin{array}{c}\text { Total } \\
\mathrm{N} \%\end{array}$} & \multicolumn{2}{|c|}{$\begin{array}{c}\text { Available } \\
\text { ppm }\end{array}$} \\
\hline & & & $\mathrm{Ca}^{+2}$ & $\mathrm{Mg}^{+2}$ & $\mathrm{Na}^{+}$ & $\mathrm{K}^{+}$ & $\mathrm{CO}_{3}^{--}$ & $\mathrm{HCO}_{3}^{-}$ & $\mathrm{CL}^{-}$ & $\mathrm{SO}_{4}^{--}$ & & $\mathbf{P} \%$ & K \\
\hline Gypsum & S1 & 3.61 & 13.86 & 7.39 & 46 & 39 & - & 8.4 & 9.8 & $17 . .89$ & 0.12 & 0.46 & 320 \\
\hline sulphur & S1 & 4.10 & 14.78 & \begin{tabular}{|l|}
8.97 \\
\end{tabular} & 5.87 & 1.39 & - & & 14.0 & $19 . .8$ & 0.12 & 0.46 & 320 \\
\hline control & S1 & 5.06 & 15.18 & 7.06 & 25.47 & 1.96 & - & 9.6 & 13.5 & $26 . .56$ & 0.11 & 0.46 & 300 \\
\hline compost & S1 & 6.98 & 21.78 & 9.57 & 36.6 & 1.86 & - & 6.9 & 42.0 & $20 . .91$ & 0.13 & 0.48 & 330 \\
\hline Gypsum & S2 & 3.47 & 15.1 & 6.73 & 11.85 & 1.03 & .. & 6.6 & 11.2 & 16.9 & 0.11 & 0.44 & 330 \\
\hline sulphur & S2 & 2.94 & 13.86 & \begin{tabular}{|l|}
6.14 \\
\end{tabular} & 8.85 & .52 & .. & 3.6 & 8.64 & 17.13 & 0.11 & 0.44 & 330 \\
\hline control & S2 & 7.31 & 25.74 & \begin{tabular}{|l|}
12.8 \\
\end{tabular} & 32.6 & 2.0 &.. & 7.2 & 42.0 & 23.94 & 0.10 & 0.40 & 280 \\
\hline compost & S2 & 7.22 & 25.08 & 12.07 & 33.2 & 1.78 & .. & 6.9 & 25.0 & 40.22 & 0.11 & 0.44 & 340 \\
\hline Gypsum & S3 & 3.39 & 11.88 & 8.58 & 11.78 & 1.63 & . & 7.9 & 14.0 & 11.96 & 0.08 & 0.40 & 320 \\
\hline sulphur & S3 & 7.30 & 23.66 & 7.92 & 39.6 & 1.86 & .. & 9.0 & 35.0 & 29.03 & 0.08 & 032 & 320 \\
\hline control & S3 & 7.21 & 22.72 & 11.16 & 36.3 & 1.86 & $\cdots$ & 7.5 & 25.0 & 39.53 & 0.07 & 033 & 290 \\
\hline compost & S3 & 8.95 & 29.04 & 17.82 & 40.6 & 2.00 &. & 13.2 & 41.6 & 34.66 & 0.09 & 0.20 & 355 \\
\hline
\end{tabular}

\section{Biological yield}

Analysis of variance showed high significantly effect of soil amendment treatments on Biological yield ton/fed in the two seasons. It could be noticed from Table4 caused a markedly positive The highest Biological yield was obtained by Giza83 and Giza35 under gypsum treatment followed by sulphur and compost comparing with control at three levels of soil salinity respectively. 
Table 4: Effect of soybean cultivars on biological yields (ton/fed) under three levels of soil salinity (means of two seasons).

\begin{tabular}{|c|c|c|c|c|c|}
\hline \multirow{3}{*}{ varieties } & \multicolumn{5}{|c|}{ \%, } \\
\hline & \multicolumn{5}{|c|}{ S1 } \\
\hline & gypsum & sulphur & compost & control & mean \\
\hline Giza111 & $3.54 \mathrm{ab}$ & $3.53 \mathrm{bc}$ & $3.40 \mathrm{ab}$ & $2.34 \mathrm{a}$ & 3.20 \\
\hline Dr 101 & $3.62 \mathrm{a}$ & $3.64 \mathrm{~b}$ & $3.36 \mathrm{ab}$ & $2.57 \mathrm{a}$ & 3.30 \\
\hline Giza35 & $3.69 \mathrm{a}$ & $3.71 \mathrm{~b}$ & $3.40 \mathrm{ab}$ & $2.45 \mathrm{a}$ & 3.19 \\
\hline Giza82 & $2.98 b$ & $3.05 c$ & $2.84 \mathrm{~b}$ & $2.45 \mathrm{a}$ & 2.83 \\
\hline Giza83 & $3.34 \mathrm{ab}$ & $3.53 \mathrm{bc}$ & $3.58 \mathrm{a}$ & $2.47 \mathrm{a}$ & 3.23 \\
\hline Toano & $3.63 \mathrm{a}$ & $3.62 \mathrm{~b}$ & $3.54 \mathrm{a}$ & $2.47 \mathrm{a}$ & 3.32 \\
\hline Holyday & $3.83 \mathrm{a}$ & $3.62 \mathrm{a}$ & $3.40 a b$ & $2.40 \mathrm{a}$ & 3.31 \\
\hline Mean & 3.52 & 3.53 & 3.36 & 2.45 & 3.21 \\
\hline \multicolumn{6}{|c|}{$\frac{1}{S 2}$} \\
\hline Giza111 & $2.89 \mathrm{~d}$ & $2.85 \mathrm{c}$ & $2.42 \mathrm{~d}$ & $2.07 \mathrm{e}$ & 2.56 \\
\hline Dr 101 & $2.91 \mathrm{c}$ & $2.8 \mathrm{e}$ & $2.37 \mathrm{e}$ & $2.04 \mathrm{f}$ & 2.53 \\
\hline Giza35 & $3.18 \mathrm{a}$ & $2.94 \mathrm{~b}$ & $2.65 \mathrm{a}$ & $2.19 \mathrm{c}$ & 2.74 \\
\hline Giza82 & $2.6 \mathrm{c}$ & $2.4 \mathrm{a}$ & $2.3 \mathrm{f}$ & $1.84 \mathrm{C}$ & 2.29 \\
\hline Giza83 & $3.02 \mathrm{~b}$ & $2.72 f$ & $2.38 \mathrm{e}$ & $2.23 \mathrm{~g}$ & 2.59 \\
\hline Toano & $3.03 \mathrm{~b}$ & $2.82 \mathrm{~d}$ & $2.45 c$ & $2.31 \mathrm{~g}$ & 2.65 \\
\hline Holyday & $3.02 \mathrm{~b}$ & $2.97 \mathrm{a}$ & $2.62 \mathrm{~b}$ & $2.12 \mathrm{~d}$ & 2.68 \\
\hline Mean & 2.95 & 2.79 & 2.46 & 2.11 & 2.58 \\
\hline \multicolumn{6}{|c|}{ s3 } \\
\hline Giza111 & $2.45 \mathrm{f}$ & $2.52 \mathrm{c}$ & $2.77 \mathrm{a}$ & $1.77 \mathrm{e}$ & 2.38 \\
\hline Dr 101 & $2.49 \mathrm{e}$ & $2.41 \mathrm{e}$ & $2.18 \mathrm{e}$ & $1.77 \mathrm{e}$ & 2.21 \\
\hline Giza35 & $2.7 \mathrm{a}$ & $2.49 d$ & $2.42 \mathrm{~b}$ & $1.89 \mathrm{c}$ & 2.38 \\
\hline Giza82 & $2.37 \mathrm{~g}$ & $2.1 \mathrm{f}$ & $2.18 \mathrm{e}$ & $1.66 \mathrm{f}$ & 2.08 \\
\hline Giza83 & $2.68 \mathrm{~b}$ & $2.4 \mathrm{e}$ & $2.2 \mathrm{~d}$ & $1.84 \mathrm{~d}$ & 2.28 \\
\hline Toano & $2.61 \mathrm{c}$ & $2.59 \mathrm{a}$ & $2.34 \mathrm{c}$ & $1.93 \mathrm{~b}$ & 2.37 \\
\hline Holyday & $2.55 d$ & $2.55 \mathrm{~b}$ & $2.33 c$ & $2.31 \mathrm{a}$ & 2.44 \\
\hline Mean & 2.55 & 2.44 & 2.35 & 1.88 & 2.30 \\
\hline
\end{tabular}

Seed yield:

Analysis of variance showed high significant effect of soil amendment treatments on seed yield in the two seasons. It could be noticed from Table (5) that gypsum treatment caused a marked positive effect on seed yield $\left(1.67,1.34\right.$ and1 1.06 tonfed $\left.^{-1}\right)$ and. (1.65.1.33and1.04) with Giza83 and Giza35 at three levels respectively, as compared with sulphur, compost and control treatment which recorded the lowest values $\left(.1 .03\right.$..83and 0.72 tonfed $^{-1)}$, at three soil salinity levels respectively with Giza82. The trend obtained for seed yield was similar to these obtained for biomass yield. Data in Table (5) show high significant effect on seed yield by soybean cultivars. The seed yield as 
affected by cultivars can be arranged as follow : Giza83 = Giza35> Giza111.>Dr101> Holyday >. Toano > Giza82) at S1. (Giza83 = Giza35> Giza111.>Dr101 > Holyday >. Toano $>$ Giza82) at S2 and Giza83 = Giza35> Giza111.>Dr101> Holyday >. Toano > Giza82) at S3 three levels of soil salinity, respectively, .This result proves that soybean Giza83 and Giza35 cultivars were superior to the others in the two seasons. The interaction between soil amendment treatments and soybean cultivars had high significant effect on soybean seed yield in both seasons. The highest seed yield was obtained by Giza83 and Giza35 under gypsum treatment followed by sulphur and compost comparing with control at three levels, of soil salinity respectively. Chaudhry (2001) also concluded that gypsum application to rice and wheat enhanced the paddy and grain yield by 18 and $17 \%$, respectively.

Table (5): Effect of soybean cultivars on seeds yields Ton/fed) under three levels of soil salinity (means of two seasons)

\begin{tabular}{|c|c|c|c|c|c|}
\hline \multirow[t]{3}{*}{ varieties } & \multicolumn{5}{|c|}{90999990999999, seed yield( ton /fed) } \\
\hline & & S1 & & & \\
\hline & gypsum & sulphur & compost & control & mean \\
\hline Giza111 & $1.67 \mathrm{a}$ & $1.56 \mathrm{~b}$ & $1.57 \mathrm{a}$ & $0.7 \mathrm{~b}$ & 1.38 \\
\hline Dr 101 & $1.66 \mathrm{a}$ & $1.6 \mathrm{a}$ & $1.52 \mathrm{~b}$ & $0.72 \mathrm{a}$ & 1.38 \\
\hline Giza35 & $1.65 a$ & $1.6 \mathrm{a}$ & $1.56 \mathrm{a}$ & $0.73 \mathrm{a}$ & 1.39 \\
\hline Giza82 & $1.03 \mathrm{c}$ & $1.01 \mathrm{c}$ & $1.01 \mathrm{~d}$ & $0.64 \mathrm{c}$ & 0.92 \\
\hline Giza83 & $1.67 \mathrm{a}$ & $1.61 \mathrm{a}$ & $1.58 \mathrm{a}$ & $0.74 \mathrm{a}$ & 1.40 \\
\hline Toano & $1.52 \mathrm{~b}$ & $1.48 \mathrm{~d}$ & $1.51 \mathrm{~b}$ & $0.73 \mathrm{a}$ & 1.31 \\
\hline Holyday & $1.64 \mathrm{a}$ & $1.46 \mathrm{~d}$ & $1.5 \mathrm{~b}$ & $0.74 \mathrm{a}$ & 1.34 \\
\hline Mean & 1.55 & 1.47 & 1.46 & 0.71 & 1.30 \\
\hline \multicolumn{6}{|c|}{ S2 } \\
\hline Giza111 & $1.12 \mathrm{c}$ & $1.09 \mathrm{ab}$ & $0.71 \mathrm{~b}$ & $0.61 \mathrm{a}$ & 0.88 \\
\hline Dr 101 & $1.14 \mathrm{c}$ & $1.07 \mathrm{ab}$ & $0.77 \mathrm{ab}$ & $0.62 \mathrm{a}$ & 0.90 \\
\hline Giza35 & $1.33 a$ & $1.13 \mathrm{a}$ & $0.83 \mathrm{a}$ & $0.63 \mathrm{a}$ & 0.98 \\
\hline Giza82 & $0.83 \mathrm{~d}$ & $0.65 \mathrm{~d}$ & $0.46 \mathrm{~d}$ & $0.42 \mathrm{~b}$ & 0.59 \\
\hline Giza83 & $1.34 \mathrm{a}$ & $1.11 \mathrm{~b}$ & $0.73 \mathrm{~b}$ & $0.64 \mathrm{a}$ & 0.96 \\
\hline Toano & $1.08 \mathrm{c}$ & $0.89 \mathrm{c}$ & $0.63 \mathrm{c}$ & $0.57 \mathrm{a}$ & 0.79 \\
\hline Holyday & $1.11 \mathrm{c}$ & $0.93 \mathrm{c}$ & $0.7 \mathrm{~b}$ & $0.56 \mathrm{a}$ & 0.83 \\
\hline Mean & 1.14 & 0.98 & 0.69 & 0.58 & 0.85 \\
\hline \multicolumn{6}{|c|}{ S3 } \\
\hline Giza111 & $0.83 \mathrm{~b}$ & $0.86 \mathrm{a}$ & $0.65 \mathrm{~b}$ & $0.43 \mathrm{bc}$ & 0.69 \\
\hline Dr 101 & $0.82 \mathrm{bc}$ & $0.77 \mathrm{~b}$ & $0.55 \mathrm{c}$ & $0.47 \mathrm{~b}$ & 0.65 \\
\hline Giza35 & $1.04 \mathrm{a}$ & $0.87 \mathrm{a}$ & $0.7 \mathrm{c}$ & $0.52 \mathrm{c}$ & 0.78 \\
\hline Giza82 & $0.72 \mathrm{~d}$ & $0.53 \mathrm{~d}$ & $0.47 \mathrm{~d}$ & $0.3 \mathrm{~d}$ & 0.51 \\
\hline Giza83 & $1.06 \mathrm{a}$ & $0.85 \mathrm{a}$ & $0.71 \mathrm{a}$ & $0.53 \mathrm{a}$ & 0.79 \\
\hline Toano & $0.79 \mathrm{c}$ & $0.77 \mathrm{~b}$ & $0.43 \mathrm{~d}$ & $0.4 \mathrm{c}$ & 0.60 \\
\hline Holyday & $0.78 \mathrm{c}$ & $0.71 \mathrm{c}$ & $0.46 \mathrm{~d}$ & $0.37 \mathrm{~d}$ & 0.58 \\
\hline Mean & 0.86 & 0.77 & 0.57 & 0.43 & 0.66 \\
\hline
\end{tabular}


straw yield:

Data in Table 6 revealed that the soybean cultivars were different significantly in straw yield in the two seasons. Holyday, Toano and Giza35 cultivars had the highest values $(2.19,2.11$ and 2.04$),(1.91,1.95$ and.1.85.) and $(1.77,1.82$ and 1.70$)$ ton/fed at three soil salinity levels respectively with gypsum treatment and the lowest values of $(1.67,1.68$ and 1.62 ton/fed) were recorded with giza83 cultivar at three soil salinity levels respectively. These differences may be due to the differences in the genetic structure of the used cultivars which led to different responses salinity and soil amendment treatments

Table 6: Effect of soybean cultivars on straw yield (ton/fed) under three levels of soil salinity (means of two seasons)

\begin{tabular}{|l|c|c|c|c|c|}
\hline \multirow{2}{*}{ varieties } & \multicolumn{5}{|c|}{ S1 } \\
\cline { 2 - 6 } & gypsum & sulphur & compost & control & mean \\
\cline { 2 - 6 } & $1.87 \mathrm{e}$ & $1.97 \mathrm{~d}$ & $1.83 \mathrm{c}$ & $1.64 \mathrm{c}$ & 1.83 \\
\hline Giza111 & $1.96 \mathrm{~d}$ & $2.04 \mathrm{c}$ & $1.84 \mathrm{c}$ & $1.85 \mathrm{a}$ & 1.92 \\
\hline Giza35 & $2.04 \mathrm{c}$ & $2.11 \mathrm{~b}$ & $1.85 \mathrm{c}$ & $1.72 \mathrm{~b}$ & 1.93 \\
\hline Giza82 & $1.95 \mathrm{~d}$ & $2.04 \mathrm{c}$ & $1.84 \mathrm{c}$ & $1.81 \mathrm{a}$ & 1.91 \\
\hline Giza83 & $1.67 \mathrm{f}$ & $1.92 \mathrm{e}$ & $1.83 \mathrm{c}$ & $1.73 \mathrm{~b}$ & 1.79 \\
\hline Toano & $2.11 \mathrm{~b}$ & $2.14 \mathrm{ab}$ & $2.1 \mathrm{a}$ & $1.74 \mathrm{~b}$ & 2.02 \\
\hline Holyday & $2.19 \mathrm{a}$ & $2.16 \mathrm{a}$ & $2.03 \mathrm{~b}$ & $1.66 \mathrm{c}$ & 2.00 \\
\hline Mean & 1.97 & 2.05 & 1.88 & 1.74 & 1.91 \\
\hline \multicolumn{5}{|c|}{ S2 } \\
\hline Giza111 & $1.77 \mathrm{~d}$ & $1.76 \mathrm{~d}$ & $1.71 \mathrm{c}$ & $1.46 \mathrm{~d}$ & 1.68 \\
\hline Dr 101 & $1.77 \mathrm{~d}$ & $1.73 \mathrm{e}$ & $1.7 \mathrm{c}$ & $1.42 \mathrm{e}$ & 1.66 \\
\hline Giza35 & $1.85 \mathrm{dc}$ & $1.81 \mathrm{c}$ & $1.82 \mathrm{~b}$ & $1.52 \mathrm{c}$ & 1.75 \\
\hline Giza82 & $1.77 \mathrm{~d}$ & $1.75 \mathrm{de}$ & $1.84 \mathrm{~b}$ & $1.42 \mathrm{e}$ & 1.70 \\
\hline Giza83 & $1.68 \mathrm{e}$ & $1.67 \mathrm{f}$ & $1.65 \mathrm{~d}$ & $1.39 \mathrm{e}$ & 1.60 \\
\hline Toano & $1.95 \mathrm{a}$ & $1.93 \mathrm{~b}$ & $1.91 \mathrm{a}$ & $1.74 \mathrm{a}$ & 1.88 \\
\hline Holyday & $1.91 \mathrm{~b}$ & $2.04 \mathrm{a}$ & $1.92 \mathrm{a}$ & $1.56 \mathrm{~b}$ & 1.86 \\
\hline Mean & 1.81 & 1.81 & 1.79 & 1.50 & 1.73 \\
\hline \multicolumn{7}{|c|}{ S3 } \\
\hline Giza111 & $1.62 \mathrm{~d}$ & $1.66 \mathrm{~b}$ & $1.62 \mathrm{c}$ & $1.34 \mathrm{~cd}$ & 1.56 \\
\hline Dr 101 & $1.67 \mathrm{c}$ & $1.64 \mathrm{~b}$ & $1.63 \mathrm{c}$ & $1.3 \mathrm{~d}$ & 1.56 \\
\hline Giza35 & $1.66 \mathrm{c}$ & $1.62 \mathrm{~b}$ & $1.72 \mathrm{~b}$ & $1.37 \mathrm{c}$ & 1.59 \\
\hline Giza82 & $1.65 \mathrm{~cd}$ & $1.57 \mathrm{c}$ & $1.71 \mathrm{~b}$ & $1.36 \mathrm{c}$ & 1.57 \\
\hline Giza83 & $1.62 \mathrm{~d}$ & $1.55 \mathrm{c}$ & $1.51 \mathrm{~d}$ & $1.31 \mathrm{~d}$ & 1.50 \\
\hline Toano & $1.82 \mathrm{a}$ & $1.82 \mathrm{a}$ & $1.91 \mathrm{a}$ & $1.53 \mathrm{a}$ & 1.77 \\
\hline Holyday & $1.77 \mathrm{~b}$ & $1.84 \mathrm{a}$ & $1.87 \mathrm{a}$ & $1.44 \mathrm{~b}$ & 1.73 \\
\hline Mean & 1.69 & 1.67 & 1.71 & 1.38 & 1.61 \\
\hline
\end{tabular}

\section{0-seed weight:}

Data in Table (7) revealed that the soybean cultivars were different significant 100-seed weight(g) in the two seasons Giza35 cultivar had the highest values $14.27,14.17,14.33$ and $13.17 \mathrm{~g}, 13.03,12.07,11.47$ and 10.17 and $12.63,12.40,11.50$ and $11.40 \mathrm{~g}$ under $\mathrm{S} 1, \mathrm{~S} 2$ and $\mathrm{S} 3$, treatment, respectively,While genotype Toano had the lowest values.1.2.11.7.11.17and 10.8.9.38.9.2.9.02and $8.93 \mathrm{~g} .9 .06 .8 .73 .8 .50$ and $8.33 \mathrm{~g}$ underS1.S2and S3 
treatment, respectively .Data in Table (7) showed that gypsum treatment caused markedly positive effect on 100-seed weight( $\mathrm{g}$ ) at three levels of soil salinity respectively followed by sulphur and compost.

Table 7: Effect of soybean cultivars on -100 seed $(\mathrm{g})$ under three levels of soil salinity (means of two seasons)

\begin{tabular}{|l|c|c|c|c|c|}
\hline \multirow{2}{*}{ varieties } & \multicolumn{5}{|c|}{ weight 100-seed( g) } \\
\cline { 2 - 6 } & gypsum & sulphur & compost & control & mean \\
\cline { 2 - 6 } & $13 \mathrm{c}$ & $12.87 \mathrm{c}$ & $13.03 \mathrm{c}$ & $10.87 \mathrm{e}$ & 12.44 \\
\hline Giza111 & $14.9 \mathrm{a}$ & $14.76 \mathrm{a}$ & $15.00 \mathrm{a}$ & $13.37 \mathrm{a}$ & 14.83 \\
\hline Giza35 & $14.27 \mathrm{~b}$ & $14.17 \mathrm{~b}$ & $14.33 \mathrm{~b}$ & $13.17 \mathrm{~b}$ & 14.27 \\
\hline Giza82 & $10.4 \mathrm{~g}$ & $10.30 \mathrm{~g}$ & $10.40 \mathrm{~g}$ & $10.10 \mathrm{f}$ & 10.40 \\
\hline Giza83 & $11.6 \mathrm{e}$ & $11.37 \mathrm{e}$ & $11.47 \mathrm{e}$ & $11.03 \mathrm{~d}$ & 11.60 \\
\hline Toano & $11.2 \mathrm{f}$ & $11.07 \mathrm{f}$ & $11.17 \mathrm{f}$ & $10.80 \mathrm{e}$ & 11.20 \\
\hline Holyday & $12.07 \mathrm{~d}$ & $11.97 \mathrm{~d}$ & $12.17 \mathrm{~d}$ & $11.20 \mathrm{c}$ & 12.07 \\
\hline Mean & 12.49 & 13.82 & 13.03 & 10.87 & 12.40 \\
\hline \multicolumn{5}{|c|}{$\mathrm{S} 2$} \\
\hline Giza111 & $12.4 \mathrm{~b}$ & $12.03 \mathrm{~b}$ & $11.03 \mathrm{~b}$ & $10.1 \mathrm{bc}$ & 11.39 \\
\hline Dr 101 & $13.17 \mathrm{a}$ & $12.80 \mathrm{a}$ & $10.9 \mathrm{~b}$ & $10.43 \mathrm{a}$ & 12.04 \\
\hline Giza35 & $13.03 \mathrm{a}$ & $12.07 \mathrm{~b}$ & $11.47 \mathrm{~g}$ & $10.17 \mathrm{ab}$ & 11.69 \\
\hline Giza82 & $9.93 \mathrm{a}$ & $9.63 \mathrm{e}$ & $9.03 \mathrm{~d}$ & $9.06 \mathrm{~d}$ & 9.41 \\
\hline Giza83 & $10.80 \mathrm{~d}$ & $10.46 \mathrm{~d}$ & $10.23 \mathrm{c}$ & $10.03 \mathrm{bc}$ & 10.50 \\
\hline Toano & $9.38 \mathrm{f}$ & $9.20 \mathrm{f}$ & $9.02 \mathrm{~d}$ & $8.93 \mathrm{~d}$ & 9.13 \\
\hline Holyday & $11.19 \mathrm{c}$ & $11.03 \mathrm{c}$ & $10.4 \mathrm{c}$ & $9.83 \mathrm{c}$ & 10.61 \\
\hline Mean & 11.41 & 10.74 & 10.30 & 9.62 & 10.68 \\
\hline \multicolumn{5}{|c|}{$\mathrm{S3}$} \\
\hline Giza111 & $11.83 \mathrm{~b}$ & $11.56 \mathrm{~b}$ & $10.30 \mathrm{~b}$ & $10.17 \mathrm{~b}$ & 10.97 \\
\hline Dr 101 & $10.83 \mathrm{c}$ & $10.40 \mathrm{c}$ & $10.23 \mathrm{~b}$ & $9.86 \mathrm{c}$ & 10.33 \\
\hline Giza35 & $12.63 \mathrm{a}$ & $12.40 \mathrm{a}$ & $11.50 \mathrm{a}$ & $11.40 \mathrm{a}$ & 11.98 \\
\hline Giza82 & $9.63 \mathrm{e}$ & $9.50 \mathrm{e}$ & $9.27 \mathrm{~d}$ & $9.13 \mathrm{~d}$ & 9.38 \\
\hline Giza83 & $10.26 \mathrm{~d}$ & $10.17 \mathrm{~d}$ & $10.01 \mathrm{c}$ & $9.90 \mathrm{c}$ & 10.09 \\
\hline Toano & $9.06 \mathrm{f}$ & $8.73 \mathrm{f}$ & $8.50 \mathrm{e}$ & $8.33 \mathrm{c}$ & 8.66 \\
\hline Holyday & $10.26 \mathrm{~d}$ & $10.17 \mathrm{~d}$ & $10.06 \mathrm{c}$ & $10.10 \mathrm{c}$ & 10.12 \\
\hline Mean & 10.64 & 10.42 & 9.98 & 9.83 & 10.22 \\
\hline
\end{tabular}

\section{Harvest index \%:}

Data in Table(8) revealed that the soybean cultivars were different significant effect on harvest index\% .The highest_Harvest index \% was obtained by Giza83 and Giza35 under gypsum treatment followed by sulphur and compost comparing with control at three levels of salinity, respectively. 
Table (8) harvest index as affected by salinity level, amendment type and soybean varieties

\begin{tabular}{|c|c|c|c|c|c|c|c|c|c|c|c|c|}
\hline \multirow[b]{2}{*}{ 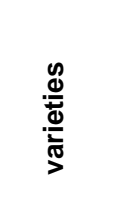 } & \multicolumn{4}{|c|}{ S1 } & \multicolumn{4}{|c|}{ S2 } & \multicolumn{4}{|c|}{ S3 } \\
\hline & 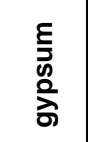 & 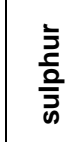 & 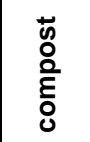 & 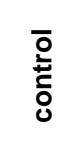 & 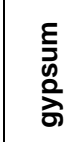 & 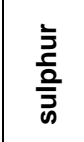 & $\begin{array}{l}\text { "ू } \\
\text { 을 } \\
\text { 엉 }\end{array}$ & 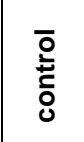 & 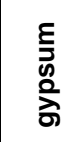 & $\frac{\vdots}{\frac{\vdots}{\frac{1}{2}}}$ & 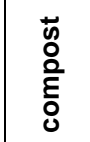 & $\begin{array}{l}\overline{\overline{2}} \\
\text { 힝 }\end{array}$ \\
\hline Giza111 & 47.17 & 44.19 & 46.17 & 29.91 & 38.75 & 38.24 & 29.33 & 29.46 & 33.87 & 34.12 & 23.46 & 24.29 \\
\hline Dr 101 & 45.85 & 43.95 & 45.23 & 28.51 & 39.17 & 38.21 & 31.17 & 30.39 & 32.93 & 31.95 & 25.22 & 26.55 \\
\hline Giza35 & 44.17 & 43.12 & 45.88 & 29.79 & 41.83 & 38.43 & 31.32 & 28.76 & 38.51 & 34.93 & 28.92 & 27.51 \\
\hline Giza82 & 34.56 & 33.11 & 35.56 & 35.35 & 46.89 & 27.68 & 20.00 & 22.82 & 30.37 & 25.23 & 21.55 & 18.07 \\
\hline Giza83 & 50.00 & 45.60 & 41.34 & 29.95 & 44.37 & 38.60 & 30.67 & 36.05 & 39.55 & 35.41 & 31.98 & 28.80 \\
\hline Toano & 51.50 & 46.88 & 42.65 & 29.55 & 35.64 & 31.56 & 24.80 & 24.67 & 30.26 & 29.72 & 18.37 & 20.75 \\
\hline Holyday & 42.81 & 40.33 & 44.11 & 30.83 & 36.75 & 45.58 & 26.71 & 26.41 & 30.58 & 27.84 & 19.79 & 37.66 \\
\hline
\end{tabular}

Nitrogen content of seeds:

Soil amendment had highly significant effect on $\mathrm{N} \%$ and protein\% of soybean seeds in both seasons (Table 9).Nitrogen \% as affected by can be arranged as follow, gypsum $>$ sulphur $>$ compost $>$ control at $s_{1}$.

Table 9: Nitrogen percentage of seeds and protein content (\%) in the seeds under three levels of soil salinity(S1,S2and S3)

\begin{tabular}{|c|c|c|c|c|c|c|c|c|c|c|}
\hline \multirow{3}{*}{ 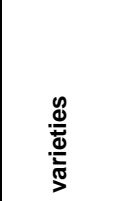 } & \multicolumn{10}{|c|}{$\% \mathrm{~N}$ seed $(\mathrm{S} 1)$} \\
\hline & \multicolumn{2}{|c|}{ gypsum } & \multicolumn{2}{|c|}{ sulphur } & \multicolumn{2}{|c|}{ compost } & \multicolumn{2}{|c|}{ control } & \multicolumn{2}{|c|}{ mean } \\
\hline & $\mathrm{N} \%$ & proten $\%$ & $\mathrm{~N} \%$ & proten $\%$ & $\mathrm{~N} \%$ & proten $\%$ & $\mathrm{~N} \%$ & proten $\%$ & $\mathrm{~N} \%$ & proten $\%$ \\
\hline Giza111 & $5.73 a$ & 32.72 & $5.83 a$ & 33.29 & $5.63 a$ & 32.15 & $4.20 \mathrm{a}$ & 23.98 & 5.35 & 30.53 \\
\hline Dr 101 & $5.63 a b$ & 32.15 & 5.70ab & 32.55 & $5.40 \mathrm{ab}$ & 30.83 & $3.60 \mathrm{bc}$ & 20.56 & 5.08 & 29.02 \\
\hline Giza35 & $5.77 a$ & 32.95 & $5.80 \mathrm{a}$ & 33.12 & $5.20 \mathrm{bc}$ & 29.69 & $3.70 \mathrm{~b}$ & 21.13 & 5.12 & 29.22 \\
\hline Giza82 & $5.33 b c$ & 30.43 & $5.40 \mathrm{bc}$ & 30.83 & 4.80de & 27.41 & 3.13de & 17.87 & 4.67 & 26.64 \\
\hline Giza83 & $5.02 \mathrm{~cd}$ & 28.66 & $5.20 \mathrm{~cd}$ & 29.69 & $5.05 \mathrm{~cd}$ & 28.84 & $3.00 \mathrm{e}$ & 17.13 & 4.57 & 26.08 \\
\hline Toano & $4.26 \mathrm{e}$ & 24.32 & $4.39 \mathrm{e}$ & 25.07 & $4.20 f$ & 23.98 & $3.30 \mathrm{cde}$ & 18.84 & 4.04 & 23.05 \\
\hline Holyday & $4.88 \mathrm{~d}$ & 27.86 & $4.91 \mathrm{~d}$ & 28.04 & $4.60 \mathrm{e}$ & 26.27 & 3.43bcd & 19.59 & 4.46 & 25.44 \\
\hline Mean & 5.23 & 29.87 & 5.32 & 30.37 & 4.98 & 28.45 & 3.48 & 19.87 & 4.75 & 27.14 \\
\hline \multicolumn{11}{|c|}{ S2 } \\
\hline Giza111 & $4.32 \mathrm{bc}$ & 24.67 & $4.40 \mathrm{bc}$ & 25.12 & $4.16 \mathrm{~b}$ & 23.75 & $3.13 a b c$ & 17.87 & 4.00 & 22.85 \\
\hline Dr 101 & $4.24 \mathrm{bc}$ & 24.21 & $4.20 \mathrm{bc}$ & 23.98 & $4.06 \mathrm{~b}$ & 23.18 & $3.33 a$ & 19.01 & 3.96 & 22.60 \\
\hline Giza35 & $4.50 \mathrm{~b}$ & 25.70 & $4.46 \mathrm{bc}$ & 25.47 & $4.00 \mathrm{~b}$ & 22.84 & $3.16 a b$ & 18.04 & 4.03 & 23.01 \\
\hline Giza82 & $4.86 a$ & 27.75 & $4.83 a$ & 27.58 & $4.63 a$ & 26.44 & $2.93 \mathrm{bcd}$ & 16.73 & 4.31 & 24.62 \\
\hline Giza83 & $4.13 c$ & 23.58 & $4.40 \mathrm{bc}$ & 25.12 & $4.13 b$ & 23.58 & $2.70 \mathrm{~d}$ & 15.42 & 3.84 & 21.93 \\
\hline Toano & $4.03 c$ & 23.01 & $4.16 \mathrm{c}$ & 23.75 & $3.96 \mathrm{~b}$ & 22.61 & $2.80 \mathrm{~cd}$ & 15.99 & 3.74 & 21.34 \\
\hline Holyday & $4.50 \mathrm{~b}$ & 25.70 & 4.53ab & 25.87 & $4.30 \mathrm{~b}$ & 24.55 & \begin{tabular}{|l|}
$2.79 \mathrm{~cd}$ \\
\end{tabular} & 15.93 & 4.03 & 23.01 \\
\hline Mean & 4.37 & 24.94 & 4.43 & 25.27 & 4.18 & 23.85 & 2.98 & 17.00 & 3.99 & 22.77 \\
\hline \multicolumn{11}{|c|}{ S3 } \\
\hline Giza111 & $4.12 a b$ & 23.53 & $4.23 b$ & 24.15 & 4.03ab & 23.01 & $2.80 \mathrm{abc}$ & 15.99 & 3.80 & 21.67 \\
\hline Dr 101 & $4.11 \mathrm{ab}$ & 23.47 & $4.10 \mathrm{bc}$ & 23.41 & $3.86 a b c$ & 22.04 & $2.93 a$ & 16.73 & 3.75 & 21.41 \\
\hline Giza35 & $4.16 a b$ & 23.75 & $4.66 \mathrm{a}$ & 26.61 & $3.63 c$ & 20.73 & $2.86 a b$ & 16.33 & 3.83 & 21.86 \\
\hline Giza82 & $4.20 \mathrm{a}$ & 23.98 & $4.26 \mathrm{~b}$ & 24.32 & $4.06 a$ & 23.18 & $2.76 a b c$ & 15.76 & 3.82 & 21.81 \\
\hline Giza83 & $4.06 a b$ & 23.18 & $4.03 \mathrm{bc}$ & 23.01 & $3.70 \mathrm{bc}$ & 21.13 & $2.50 c$ & 14.28 & 3.57 & 20.40 \\
\hline Toano & $3.83 \mathrm{bc}$ & 21.87 & $4.03 \mathrm{bc}$ & 23.01 & 3.73abc & 21.30 & $2.56 \mathrm{bc}$ & 14.62 & 3.54 & 20.20 \\
\hline Holyday & $3.63 c$ & 20.73 & $3.80 c$ & 21.70 & $3.53 c$ & 20.16 & $2.48 c$ & 14.16 & 3.36 & 19.19 \\
\hline Mean & 4.02 & 22.93 & 4.16 & 23.75 & 3.79 & 21.65 & 2.70 & 15.41 & 3.67 & 20.93 \\
\hline
\end{tabular}




\section{Phosphorus content of seeds:}

Data in (Table10) show that a high significant effect of soil amendments treatments on P-content of soybean seeds in the two seasons can be arranged in decreasing order as follow, gypsum>sulphur > compost > control.

Table 10:phosphrous percentage (\%) of seeds under three levels of soil salinity (S1, S2and S3)

\begin{tabular}{|c|c|c|c|c|c|}
\hline \multirow[t]{3}{*}{ varieties } & \multicolumn{5}{|c|}{ seed $\mathrm{P} \%$} \\
\hline & gypsum & sulphur & compost & control & mean \\
\hline & \multicolumn{5}{|c|}{$\mathrm{S} 1$} \\
\hline Giza111 & $0.61 \mathrm{~cd}$ & $0.61 \mathrm{c}$ & $0.52 \mathrm{c}$ & $0.45 b$ & 0.55 \\
\hline Dr 101 & $0.68 a$ & $0.61 c$ & $0.51 c$ & $0.40 \mathrm{~d}$ & 0.55 \\
\hline Giza35 & $0.60 d$ & $0.63 b$ & $0.62 a$ & $0.42 c$ & 0.57 \\
\hline Giza82 & $0.65 b$ & $0.61 \mathrm{c}$ & $0.61 a$ & $0.41 \mathrm{~cd}$ & 0.57 \\
\hline Giza83 & $0.69 a$ & $0.66 a$ & $0.43 d$ & $0.42 c$ & 0.55 \\
\hline Toano & $0.51 \mathrm{e}$ & $0.47 d$ & $0.37 e$ & $0.31 \mathrm{e}$ & 0.42 \\
\hline Holyday & $0.62 c$ & $0.61 c$ & $0.57 b$ & $0.47 a$ & 0.57 \\
\hline Mean & 0.62 & 0.60 & 0.52 & 0.41 & 0.54 \\
\hline \multicolumn{6}{|c|}{ S2 } \\
\hline Giza111 & $0.58 a$ & $0.50 \mathrm{~b}$ & $0.50 a$ & $0.49 a$ & 0.52 \\
\hline Dr 101 & $0.57 a$ & $0.51 \mathrm{ab}$ & $0.41 c$ & $0.38 c$ & 0.47 \\
\hline Giza35 & $0.52 b$ & $0.52 a b$ & $0.41 c$ & $0.34 d$ & 0.42 \\
\hline Giza82 & $0.53 b$ & $0.50 \mathrm{ab}$ & $0.46 b$ & $0.44 b$ & 0.48 \\
\hline Giza83 & $0.50 c$ & $0.48 c$ & $0.40 c$ & $0.38 c$ & 0.44 \\
\hline Toano & $0.42 e$ & $0.40 d$ & $0.40 c$ & $0.28 \mathrm{e}$ & 0.38 \\
\hline Holyday & $0.45 d$ & $0.41 d$ & $0.40 c$ & $0.38 \mathrm{c}$ & 0.41 \\
\hline Mean & 0.51 & 0.47 & 0.43 & 0.38 & 0.45 \\
\hline \multicolumn{6}{|c|}{ S3 } \\
\hline Giza111 & $0.47 b$ & $0.42 c$ & $0.41 b$ & $0.29 b$ & 0.40 \\
\hline Dr 101 & $0.47 b$ & $0.41 b c$ & $0.35 \mathrm{e}$ & $0.31 a$ & 0.39 \\
\hline Giza35 & $0.33 d$ & $0.32 e$ & $0.38 d$ & $0.28 b$ & 0.33 \\
\hline Giza82 & $0.46 b$ & $0.43 b$ & $0.42 b c$ & $0.24 c$ & 0.39 \\
\hline Giza83 & $0.50 a$ & $0.51 \mathrm{a}$ & $0.45 a$ & $0.31 \mathrm{a}$ & 0.44 \\
\hline Toano & $0.46 b$ & $0.41 \mathrm{c}$ & $0.39 \mathrm{~cd}$ & $0.23 c$ & 0.37 \\
\hline Holyday & $0.42 c$ & $0.37 d$ & $0.31 f$ & $0.29 \mathrm{~b}$ & 0.35 \\
\hline Mean & 0.44 & 0.41 & 0.39 & 0.28 & 0.38 \\
\hline
\end{tabular}

\section{Potassium content of seeds:}

The results in (Table 11) show a highly significant effect of soil amendment treatments on $\mathrm{K}$ content in seeds in the two seasons. The highest values gypsum $>$ sulphur $>$ compost $>$ control at $s_{1}$, respectively 
Table (11):potassium percentage (\%) of seeds under three levels of soil salinity

\begin{tabular}{|l|c|c|c|c|c|}
\hline \multirow{2}{*}{ varieties } & \multicolumn{5}{|c|}{ seed K\% } \\
\cline { 2 - 6 } & gypsum & sulphur & compost & control & mean \\
\cline { 2 - 6 } & \multicolumn{5}{|c|}{ S1 } \\
\hline Giza111 & $1.7 \mathrm{ab}$ & $1.72 \mathrm{ab}$ & $1.65 \mathrm{a}$ & $1.13 \mathrm{a}$ & 1.55 \\
\hline Dr 101 & $1.63 \mathrm{bc}$ & $1.70 \mathrm{bc}$ & $1.61 \mathrm{a}$ & $0.94 \mathrm{~b}$ & 1.47 \\
\hline Giza35 & $1.74 \mathrm{a}$ & $1.78 \mathrm{a}$ & $1.68 \mathrm{a}$ & $0.95 \mathrm{~b}$ & 1.54 \\
\hline Giza82 & $1.65 \mathrm{bc}$ & $1.63 \mathrm{c}$ & $1.41 \mathrm{c}$ & $0.83 \mathrm{c}$ & 1.38 \\
\hline Giza83 & $1.61 \mathrm{c}$ & $1.65 \mathrm{bc}$ & $1.48 \mathrm{bc}$ & $0.85 \mathrm{c}$ & 1.40 \\
\hline Toano & $1.65 \mathrm{bc}$ & $1.64 \mathrm{bc}$ & $1.42 \mathrm{c}$ & $0.70 \mathrm{~d}$ & 1.35 \\
\hline Holyday & $1.63 \mathrm{bc}$ & $1.65 \mathrm{bc}$ & $1.50 \mathrm{~b}$ & $0.71 \mathrm{~d}$ & 1.37 \\
\hline Mean & 1.66 & 1.68 & 1.54 & 0.87 & 1.44 \\
\hline \multicolumn{5}{|c|}{$\mathrm{S} 2$} \\
\hline Giza111 & $1.63 \mathrm{a}$ & $1.64 \mathrm{ab}$ & $1.51 \mathrm{a}$ & $0.86 \mathrm{a}$ & 1.41 \\
\hline Dr 101 & $1.60 \mathrm{a}$ & $1.65 \mathrm{ab}$ & $1.44 \mathrm{bc}$ & $0.85 \mathrm{a}$ & 1.39 \\
\hline Giza35 & $1.65 \mathrm{a}$ & $1.66 \mathrm{a}$ & $1.49 \mathrm{ab}$ & $0.81 \mathrm{ab}$ & 1.40 \\
\hline Giza82 & $1.63 \mathrm{a}$ & $1.58 \mathrm{bc}$ & $1.48 \mathrm{c}$ & $0.78 \mathrm{~b}$ & 1.37 \\
\hline Giza83 & $1.50 \mathrm{~b}$ & $1.55 \mathrm{c}$ & $1.37 \mathrm{c}$ & $0.60 \mathrm{~b}$ & 1.26 \\
\hline Toano & $1.48 \mathrm{~b}$ & $1.58 \mathrm{bc}$ & $1.37 \mathrm{c}$ & $0.61 \mathrm{c}$ & 1.26 \\
\hline Holyday & $1.45 \mathrm{~b}$ & $1.52 \mathrm{c}$ & $1.29 \mathrm{~d}$ & $0.57 \mathrm{c}$ & 1.21 \\
\hline Mean & 1.56 & 1.60 & 1.42 & 0.73 & 1.33 \\
\hline \multicolumn{7}{|c|}{$\mathrm{S} 3$} \\
\hline Giza111 & $1.2 \mathrm{bc}$ & $1.32 \mathrm{a}$ & $1.29 \mathrm{a}$ & $0.74 \mathrm{~b}$ & 1.14 \\
\hline Dr 101 & $1.16 \mathrm{bcd}$ & $1.35 \mathrm{a}$ & $1.13 \mathrm{~cd}$ & $1.12 \mathrm{a}$ & 1.19 \\
\hline Giza35 & $1.31 \mathrm{a}$ & $1.30 \mathrm{ab}$ & $1.20 \mathrm{bc}$ & $0.73 \mathrm{~b}$ & 1.14 \\
\hline Giza82 & $1.23 \mathrm{~b}$ & $1.21 \mathrm{c}$ & $1.21 \mathrm{~b}$ & $0.72 \mathrm{~b}$ & 1.09 \\
\hline Giza83 & $1.16 \mathrm{bcd}$ & $1.23 \mathrm{bc}$ & $0.93 \mathrm{e}$ & $0.56 \mathrm{c}$ & 0.97 \\
\hline Toano & $1.10 \mathrm{~d}$ & $1.23 \mathrm{bc}$ & $0.92 \mathrm{e}$ & $0.53 \mathrm{~cd}$ & 0.95 \\
\hline Holyday & $1.13 \mathrm{~d}$ & $1.25 \mathrm{bc}$ & $1.06 \mathrm{~d}$ & $0.49 \mathrm{~d}$ & 0.98 \\
\hline Mean & 1.18 & 1.27 & 1.11 & 0.70 & 1.06 \\
\hline
\end{tabular}

\section{CONCLUSION}

IT can be concluded that the Giza 35 and Giza83 varieties were tolerant to soil salinity. Application of gypsum was found to be effective amendment for improving salt affected soils

Not only increase the yield of soybean but also improve soil properties which it reduces SAR value under more frequent of irrigation and with effective drainage which it allows leaching of salts out root zone

\section{REFERENCES}

Abd-Allah, M. A. A. (1998). Influence of sulphur and organic manures application on yield of sugar beet, subsequent crops \& some chemical properties of salt affected soils. (Ph.D.) Thesis, Fac. of Agric., Kafr EL-Sheikh. Tanta Univ. Egypt.

Agrisent: Manures http(2011),/ sikkimagrisent.org/general/en/manuresfertilizers aspx.printertion 12/12/2011 B.A. Stewart and D.R. Nielsen. 
Ahmad, M., N. Husain, M. Salim and B.H. Niazi. (2001) Use of chemical amendments for reclamation ofsaline-sodic soils. Int'l. J. Agri. Biol. 3: 305-307

Ahmad, M., N. Husain, MSalim and B.H. Niazi.. 2001.- Use of chemical amendments for reclamation ofsaline-sodic soils. Int'l. J. Agri. Biol. 3: 305307.

Ahmed A.M., M.M. Heikal and M.A. Shaddad (1983). Changes in growth; photosynthesis and fat content of some oil- producing plant over a range of salinity stress. Acta Agron. 32, 370-375.

Ayers, R.S.,and Westcot, D.W., (1985).Water quality for agriculture. FAO Irrigation and Drainage Paper 29, Rev. 1. FAO, Rome, 63 pp

Biggar, J.W. (1996). Regional salinity/sodicity issue inPunjab, Pakistan. Report IWMI, Lahore,

Bresler, E.B., B.L. McNeal and D.L. Carter. (1982). Salineand Sodic soils, Principles-dynamic-modeling.

Chaudhry, M.R. (2001). Gypsum efficiency in the amelioration of saline-sodic /sodic soils. Int'l. J.Agric. Biol. 3: 276-280.

Drossopoulos J.B.; A.J. Karamanos and C.A. Niavis (1987). Changes in ethanol Soluble Carobhydrates during the development of two cultivars subjected to different degrees of water stress. Ann. Bot. 59, 173-180.

F.A.O/WHO (1973). Committee on Energy and Protein

Haq, I., S.G. Khattak, H. Rahman, A. Ali and M. Salim.2001. Effect of various amendments on yield ofrice crop under saline-sodic conditions in Mardan/Swabi districts. Int'l. J. Agric. Biol. 3:289-291.

Hasegawa, P. M.; Bressan, P. A.; Zhu, J.; Bohnert, H. J. (2000). Plant cellularand molecular responses to high salinity. Annu. Rev. PlantPhysiol. Plant Mol. Biol. 51: 463-499;. doi: 10.1146/ annurev. arplant. 51.1.463.

Hassan, G., M. Sadiq, M. Jamil, S.M. Mehdi and A. Sattar(2011) |.infilNovember-December 2011 Crops \& Soils magazine 9 AmendingJ. Agric. Biol. 3: 326-332.

Jackson, M. L. (1967) "Soil Chemical Analysis "Pretic Hall India Part.I.E d.,New Delhi,India.

Jefferies, R. L. (1988). Halophytes unity and diversity in response to salinity. International symposium on physiological Ecology of Aquatic plant. Aarhus, Denmark.

Khan, G.S. 1998. Soil salinity/sodicity status in PakistanSoil survey of Pakistan, Lahore, Pakistan 59 p.

Klute, A. (1986). Methods of Soil Analysis. Part1: Physical and mineralogical methods ( $2^{\text {nd }}$ Ed.) Am. Soc. Agron. In Soil Sci. Soc. Am. Inc. Madison. Wisconsim, USA.

Knany,R.E; A.M.Masoud and Y.B.EL-Waraky(2004). Comparative study between bio fertilization and sulphur on availability of added phosphorus to faba bean plants under high $\mathrm{pH}$ soil conditions.J.Agric.Sci.Mansoura Univ., 29 (8):4801- 4809

Mardan/Swabi districts. Int'l. J. Agric. Biol. 3:289-291. 
Mohamed, Amal.A.; E.N. Gendy; KH.A. Asslely and Kh.B.M. Salem(2001). The effect of sulphur and manganese foliar application on lupine (lupinus terms L.) yield and its main component" J.Agric. Sci. Mansoura Univ., 26(1): 529.

Muhammad, S.( 1990). Salt affected and water logged soils in Pakistan. An overview. Soil Salinity and Water Management vol. (1). Proc. of IndoPak Workshop on Soil salinity and WaterManagement, Feb. 10-14, (1990), Islamabad,Pakistan pp. 21-37.p.

Page, A. L. (1982). Methods of Soil Analysis. Part 2: chemical and microbial properties (2 ${ }^{\text {nd }}$ Ed.)Am.Soc. Agron.in Soil Sci.Soc. Am.Inc .Madison. Wisconsin, USA.

Pakistan. pp. 1-26.

Rangarajan, A.; W. Brinton; A. Gabrial; L. Tivers and B. Calowell (2000). Organic Farming Research Foundation Project Report, Sarta Cruz, California, USA.

Rechcigl, J. E. (1995). Soil Amendments and Environmental Quality, CRC. Press, Inc.

Rhoades, J.D. and J. Loveday. (1990). Salinity in irrigatedagriculture. In: (ed.), Irrigation of Agriculture Crops, USACSSAAgro. No.31, Madison, WI. pp. 1084-1142.

Rhoades, J.D. and J. Loveday. (1990.) Salinity in irrigatedagriculture. In: B.A. Stewart and D.R. Nielsen(ed.), Irrigation of Agriculture Crops, USACSSAAgro. No.31, Madison, WI. pp. 1084-1142.

rice crop under saline-sodic conditions in

Sabir Gul Khattak et al. Effect of various levels of gypsum application on salt affected saline-sodic soils. Int'l. J. Agri. Biol. 3: 305-307.

Shannon M (1984). Breeding selection and the geneties of salt tolerance in salt tolerance in plants. Strategies for crop improvement, eds. Staples RC. G.H toenniessen, pp : 300-308 Wiley, New York.

Sharma, R.D., S. Ali and G.B. Part.( 1996). Effect of soil amendments on rice yield. IRRI Notes, 21: 72-73.

Siddique, M.T., M. Rashid and B.A. Ehsan.( 1988). Effect and fertilizer on the yield of rice, wheatand barley crops in saline sodic soil. Proc. 1stNational Cong. Soil Sci, Oct. 1985, Lahore. pp.380-385.

Siddique, M.T., M. Rashid and B.A. Ehsan.(1988). Effect of gypsum and fertilizer on the yield of rice, wheat and barley crops in saline sodic soil. Proc. 1stNational Cong. Soil Sci, Oct. 1985, Lahore. pp.380-385.

Singh. N.T., G.S. Hira and M.S. Bajwa. (1981). Use of amendments in reclamation of alkali soils inIndia. Agro. Kemia ES, Talajtan Tom. 30.Supplement, 158-177.

Snedecor,G.W. and W.G. Cochran(1980). Statistical Methods $.7^{\text {th }}$ Ed.225-330 .Lowa state Univ.,Press.,Ames, Lowa. USA

Springer-Verlag, NY, USA. 236 p.

Sui,Y.and M. L. Thompson (2000). Phosphorus sorption, desorption, and buffering capacity in abio-solds- amended Mohsol. Soil Sci. Soc.Am.J.64:945-954.

Tan, K. H. (Ed.) (1993). "Principles of Soil Chemistry" 2" . Marcel Dekker Inc., New York., USA. 
Verma, K.S. and I.P. Abrol. (1980). Effects of gypsum andpyrites on soil properties in a highly sodic soil.Indian J. Agric. Sci. 50: 844-851

Verma, K.S. and I.P. Abrol.(1980). Effects of gypsum and pyrites on soil properties in a highly sodic soil.Indian J. Agric. Sci. 50: 844-851.

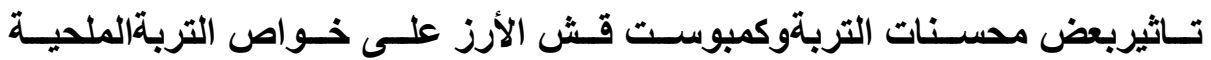

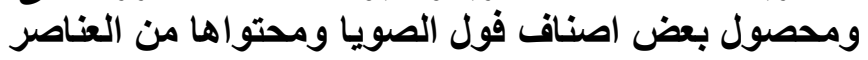

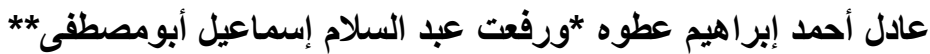

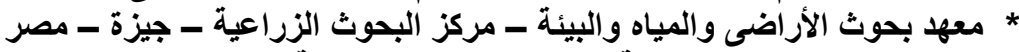

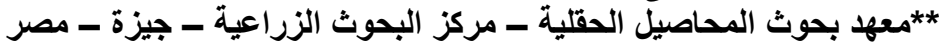

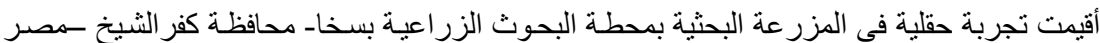

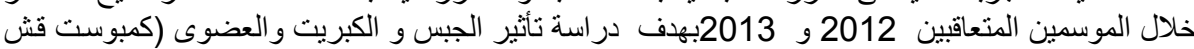

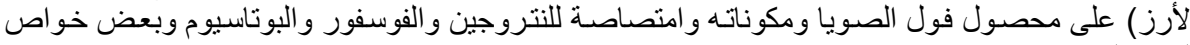

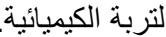

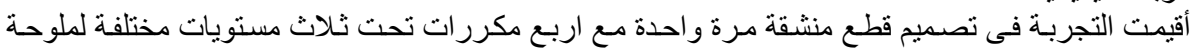
التربة وهى:

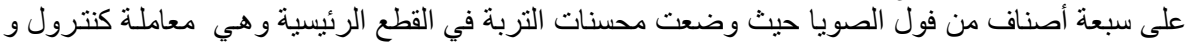

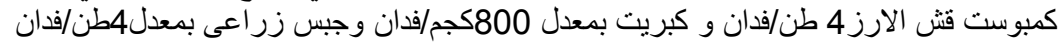

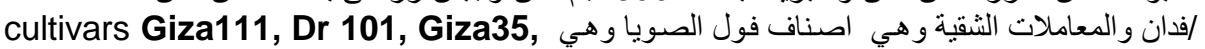
Giza82, Giza83, Toano and Holyday وتثلخص النتائج كمايلى :-19

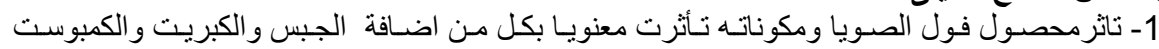

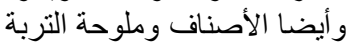
2-أعلى قيمة للمحصول الحيوى ومحصول الحبوب كانت مع اضـافة الجبس تحت صنف جيزة35 وجيزة 83

3- تحصل علي اعلي محتوي للنتروجين و الفوسفور و البوتاسيوم مـع الاصناف جيزة 35 وجيزة 83 تحت

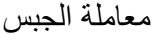
4- أانخفضت ملمة دانة التربة مع الجبس بالمقارنة مع الكبريت و ,الكمبوست و الكنترول

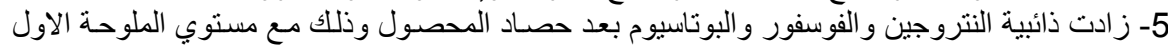

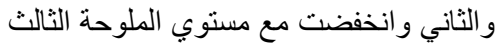

6- تحمل اصناف فول الصويا للملوحة اخذ الترتيب التاليعند مستوي الملوحة الاول

Giza83 = Giza35= Giza111=Dr101= Holyday =. Toano $>$ Giza82)

Giza83 = Giza35> Giza111.=Dr101= Holyday =. Toano > Giza82), عند and Giza83 = Giza35 Giza111.=Dr101> Holyday =. Toano > Giza82) عند المستوي الثالث تحت معاملة الجبس الزراعي 